\title{
The comparison of glucose and lipid metabolism parameters in drug-naïve, antipsychotic-treated, and antipsychotic discontinuation patients with schizophrenia
}

\section{Xiaoli Wu ${ }^{1,2}$ \\ Zeping Huang ${ }^{3}$ \\ Hongying $\mathrm{Han}^{2}$ \\ Zhiyong Zhong ${ }^{2}$ \\ Zhaoyu Gan ${ }^{2}$ \\ Xiaofeng Guo' \\ Feici Diao ${ }^{2}$ \\ Zili $\mathrm{Han}^{2}$ \\ Jingping Zhao'}

'Mental Health Institute of the Second Xiangya Hospital, Key Laboratory of Psychiatry and Mental Health of Hunan Province, Central South University, Changsha, Hunan, People's Republic of China; ${ }^{2}$ Psychiatry Department, the Third Affiliated Hospital, Sun Yat-sen University, Guangzhou, Guangdong, People's Republic of China; ${ }^{3}$ Ultrasound Department, The Third Affiliated Hospital, Sun Yat-sen University, Guangzhou, Guangdong, People's Republic of China
This article was published in the following Dove Press journal: Neuropsychiatric Disease and Treatment

22 July 2014

Number of times this article has been viewed

Background: Although many studies have reported that glucose and lipid metabolism disorders are a significant side effect associated with the use of antipsychotic drugs, the characteristics of glucose and lipid metabolism disorders in patients with schizophrenia who are taking antipsychotic drugs remain poorly understood, and the possible effects that antipsychotic discontinuation may have on glucose and lipid metabolism remain unclear.

Methods: The sample consisted of 131 Chinese patients with schizophrenia, including 70 firstepisode, drug-naïve patients; 33 patients who had received continuous antipsychotic drug treatment for $\geq 1$ year prior to the beginning of the study; and 28 patients who had discontinued antipsychotic drug treatment for $\geq 3$ months prior to the beginning of study. We compared the glucose and lipid metabolic parameter levels among the three groups of patients with schizophrenia. All assessments were performed upon hospital admission.

Results: The characteristics of glucose and lipid metabolism disorders in Chinese patients with schizophrenia who are taking antipsychotic drugs included significant augmentation of the body mass index and waist circumference, significantly higher levels of fasting plasma insulin and insulin resistance, and significantly lower plasma high-density lipoprotein cholesterol levels. Antipsychotic discontinuation appeared to not significantly improve any plasma glucose and lipid metabolic parameter levels.

Conclusion: The results suggest that antipsychotic drugs aggravate glucose and lipid metabolism disorders and that antipsychotic discontinuation is generally not associated with improvements in the parameters that indicate glucose and lipid metabolism disorders in Chinese patients with schizophrenia.

Keywords: antipsychotic-treated, antipsychotic discontinuation, drug-naïve, glucose, lipid, schizophrenia

\section{Introduction}

The prevalence of cardiovascular disease and its mortality rate are much higher among patients with schizophrenia than among the general population, ${ }^{1-5}$ and both glucose and lipid metabolism disorders are said to be important risk factors. ${ }^{6-9}$ Since the 1990s, many studies ${ }^{10-12}$ have reported that glucose and lipid metabolic parameters worsened in schizophrenia patients who took antipsychotic drugs. However, many studies ${ }^{13-18}$ have also shown that the incidence rates of impaired glucose tolerance and type 2 diabetes are far higher among drug-naïve patients with schizophrenia and their first-grade relatives than among the general population, and similar reports ${ }^{19}$ exist in the preneuroleptic literature. Therefore, the relationship between schizophrenia and 
antipsychotic drugs and glucose and lipid metabolism disorders is still not fully understood. One of main reasons for the uncertainty is the difficulty of disentangling the interactions between schizophrenia and antipsychotic drugs in clinical observation study designs.

Although many studies ${ }^{10,20-23}$ in the past two decades have reported abnormal glucose and lipid metabolic parameters in schizophrenia patients taking antipsychotic drugs, the characteristics of glucose and lipid metabolism disorders have remained controversial. ${ }^{24-28}$ Furthermore, the potential effects of antipsychotic drug discontinuation on glucose and lipid metabolism in patients with schizophrenia remain unclear because few studies have specifically addressed that issue.

In this study we compared glucose and lipid metabolism parameters among patients with schizophrenia who were drug-naïve, those currently being treated with antipsychotic drugs, and those who had discontinued taking antipsychotic drugs. Our purpose was to illuminate the authentic effects of antipsychotic drugs on glucose and lipid metabolism parameters in Chinese populations with schizophrenia and to preliminarily explore the possible effects of antipsychotic discontinuation on glucose and lipid metabolism.

\section{Materials and methods Study participants}

All participants were of Chinese descent and were inpatients recruited through the Department of Psychiatry of the Third Affiliated Hospital of Sun Yat-Sen University, Guangzhou, Guangdong, People's Republic of China. A Diagnostic and Statistical Manual of Mental Disorders Fourth Edition (DSM-IV) diagnosis of schizophrenia was established using the structured clinical interview for DSM-IV axis I disorders, which was administered by a single experienced psychiatrist (Hongying Han) and subsequently reviewed by a senior psychiatrist (Zili Han). Of 131 total inpatients, 70 were identified as exhibiting first-episode, drug-naïve schizophrenia (drug-naïve group), 33 were schizophrenia patients who had undergone continuous antipsychotic treatment for $\geq 1$ year prior to the beginning of the study (antipsychotic-treated group), and 28 were schizophrenia patients who had received antipsychotic treatment for $\geq 1$ year but had discontinued treatment for $\geq 3$ months prior to the beginning of the study (antipsychotic discontinuation group). To confirm their schizophrenia diagnoses, all first-episode patients were followed for at least 6 months after discharge.

Participants were excluded if they had a history of recognized cardiovascular disease, diabetes mellitus, malignancy, endocrine disease, dehydration, pregnancy, high-dose estrogen therapy, treatment with lipid-lowering medications, or other mental illnesses, including mood disorders, neurosis, a comorbid DSM-IV diagnosis of alcohol or illicit drug abuse, or an eating disorder. Participants who declined to provide written informed consent or who declined any assessment were also excluded. Retrospective interviews were used to exclude treated patients who had possible glucose and lipid metabolism abnormalities at baseline.

After the participants received a complete description of the study, their written informed consent was obtained in accordance with National Health and Medical Research Council guidelines. The protocol was reviewed and approved by the ethics committee at the Third Affiliated Hospital of Sun Yat-Sen University prior to the onset of the study.

\section{Study design}

This was an open-label, cross-sectional study conducted from October 2009 to March 2012. Fasting plasma levels of glucose, insulin, C-peptide, and lipid profile parameters, including triglyceride (TG), high-density lipoprotein cholesterol (HDL-C), total cholesterol (TC), low-density lipoprotein cholesterol (LDL-C), apolipoprotein A1 (aPOA1), and apolipoprotein B100 (aPOB100), were tested at the beginning of the study in the hospital's central laboratory. To ensure that each patient fasted, we assigned two nurses to monitor the study participants. The symptom severities of all patients were rated using the Positive and Negative Syndrome Scale upon admission.

\section{Assessments}

The assessments utilized in the present analysis included body mass index (BMI), waist-hip ratio (WHR), and glucose and lipid parameters for all patients upon admission. Only the results from blood samples collected at morning fasting times were included in this analysis. Blood samples were collected from all subjects between 9 am and 10 am after a 12-hour overnight fast.

Plasma glucose measurements ( $\mathrm{mmol} / \mathrm{L})$ were determined using an enzymatic photometric test (glucose oxidase-phenol plus aminophenazone) method (DiaSys Diagnostic System GmbH, Co., Ltd, Shanghai, People's Republic of China). Plasma insulin levels (mU/L) were tested using ADVIA Centaur with direct chemiluminescence (Siemens Healthcare Diagnostics, Inc., Walpole, MA, USA). The sensitivity of the assay was $0.5 \mathrm{mU} / \mathrm{L}$. The intra- and interassay coefficients of variation were $3.3 \%-4.6 \%$ and $4.8 \%-5.9 \%$, respectively. The same method was applied to detect plasma C-peptide levels $(\mathrm{ng} / \mathrm{mL})$, and the sensitivity of the assay was $0.05 \mathrm{ng} / \mathrm{mL}$. The inter- and intra-assay coefficients of variation were $3.7 \%-4.1 \%$ and $1.0 \%-3.3 \%$, respectively. The formula for 
the homeostasis assessment model was as follows: insulin resistance index $(\mathrm{HOMA}-\mathrm{IR})=\left(\right.$ fasting insulin $\left[10^{3} \mu \mathrm{U} / \mathrm{L}\right] \times$ fasting glucose $[\mathrm{mmol} / \mathrm{L}]) / 22.5 .^{29}$

The fasting plasma levels of aPOA1 and aPOB100 were measured using the immunoturbidimetry method (Sichuan Maker Biotechnology Co., Ltd, Chengdu, People's Republic of China). The ranges of detectable plasma aPOA1 and aPOB100 levels were 0-2.00 g/L and 0-2.50 g/L, respectively. The interassay coefficients of variation were less than $5 \%$ for both aPOA1 and aPOB100. The fasting plasma levels of TC, TG, and HDL-C were analyzed enzymatically using commercially available equipment (Sichuan Maker Biotechnology Co., Ltd). The fasting plasma levels of LDL-C were calculated using the formula described by Friedewald et al. ${ }^{30}$

For all subjects, anthropometric measurements (weight, height, and waist and hip circumference) were taken after participants had removed their shoes and upper garments and donned an examination gown. Waist circumference was measured midway between the iliac crests and the costal margins, and hip circumference was measured at the widest part of the hips. The WHR was used as a measure of upper body adiposity. BMI was computed as body weight (in kilograms) divided by height squared $\left(\mathrm{m}^{2}\right)$.

\section{Statistical analysis}

SPSS for Windows (version 13.0; SPSS Inc., Chicago, IL, USA) was used to analyze all data. All observed variables but
TG, WHR, IR, and aPOB100 were in accordance with normal distribution. We transformed TG, WHR, IR, and APOB100 to their normal score by rank, and then compared these clinical variables among groups. Demographic characteristics were compared using $\chi^{2}$ tests, independent samples $t$-tests, or analyses of variance as appropriate. Age, sex, illness course, and the severity of illness were considered concomitant variables, and analysis of covariance was performed to compare BMI, WHR, glucose, and lipid metabolism parameters among groups. All statistical tests were evaluated at the 5\% significance level.

\section{Results \\ Demographic characteristics and basic descriptive data}

In total, 131 patients entered the trial, and all of their data were included in the present report. Age, sex, number of hospitalizations, course of illness, and total Positive and Negative Syndrome Scale score were not equivalent between groups, whereas education level was equivalent. However, our analysis showed that the number of hospitalizations was unrelated to BMI, WHR, waist circumference, and glucose and lipid metabolic parameters. Furthermore, the prior prescriptions of different types of antipsychotic drugs were equivalent between antipsychotic-treated and antipsychotic discontinuation patients with schizophrenia (see Table 1).

Table I Demographic characteristics and basic descriptive data

\begin{tabular}{|c|c|c|c|c|}
\hline & $\begin{array}{l}\text { Drug-naïve patients } \\
\mathrm{n}=70\end{array}$ & $\begin{array}{l}\text { Antipsychotic-treated } \\
\text { patients } \\
\mathrm{n}=33\end{array}$ & $\begin{array}{l}\text { Antipsychotic } \\
\text { discontinuation patients } \\
\mathbf{n}=\mathbf{2 8}\end{array}$ & $\begin{array}{l}F / \chi^{2} / t \\
P \text {-value }\end{array}$ \\
\hline \multicolumn{5}{|l|}{ Demographic characteristics } \\
\hline Age (years) & $24.49(6.97)$ & $28.64(8.02)$ & $28.86(8.24)$ & $5.210,0.007^{\mathrm{a}}$ \\
\hline Sex (female/male) & $37 / 33$ & $7 / 26$ & $12 / 16$ & $9.177,0.012^{b}$ \\
\hline Education (primary/high/scholar/master) & $24 / 21 / 23 / 2$ & $|2 / 9 /||| \mid$ & $13 / 9 / 6 / 0$ & $2.72,0.844$ \\
\hline \multicolumn{5}{|l|}{ Descriptive data } \\
\hline Frequency of hospitalization & & $2.97(1.40)$ & $2.07(1.15)$ & $59.11,0.000$ \\
\hline PANSS total score & $92.05(11.62)$ & $85.97(5.32)$ & $86.75(5.39)$ & $6.268,0.003^{c}$ \\
\hline Course of illness (years) & $0.50(0.02)$ & $7.09(4.42)$ & $4.85(3.68)$ & $70.01,0.000^{d}$ \\
\hline Time of discontinuation (years) & & & $0.67(0.55)$ & \\
\hline \multirow{2}{*}{\multicolumn{5}{|c|}{$\begin{array}{l}\text { Past medication use (FGAs alone, } \\
\text { SGAs alone, FGAs + SGAs) }\end{array}$}} \\
\hline & & & & \\
\hline FGAs alone & & 0 & 3 & \\
\hline SGAs alone & & 23 & 21 & 0.067 \\
\hline FGAs + SGAs & & 10 & 4 & \\
\hline
\end{tabular}

Notes: Values are means (standard deviation) unless otherwise indicated. ${ }^{a}$ The average age of both antipsychotic-treated and discontinuation patients was higher than that of drug-naïve patients $(P=0.0 \mathrm{I})$, and no significant difference was found between antipsychotic-treated and discontinuation patients $(P=0.909)$. ${ }^{b} S e x$ distribution was not equal between drug-naïve and antipsychotic-treated patients $(P=0.003)$, and sex distribution was equal between drug-naïve and antipsychotic discontinued patients $(P=0.37 \mathrm{I})$ and between antipsychotic-treated and discontinuation patients $(P=0.371)$. cPANSS total scores of both antipsychotic-treated and discontinuation patients were far higher than those of drug-naïve patients $(P=0.002, P=0.012)$, and no significant difference was found between antipsychotic-treated and discontinuation patients $(P=0.743)$. ${ }^{d}$ The course of illness was not balanced among drug-naïve, antipsychotic-treated, and discontinuation patients $(P=0.000, P=0.000, P=0.002$, respectively).

Abbreviations: FGAs, first-generation antipsychotics; PANSS, Positive and Negative Syndrome Scale; SGAs, second-generation antipsychotics. 
The comparison of BMI,WHR, and glucose and lipid metabolic parameters among drug-naïve, antipsychotic-treated, and antipsychotic-discontinuation patients with schizophrenia

Compared with first-episode, drug-naïve patients with schizophrenia, antipsychotic-treated patients with schizophrenia had much higher plasma insulin and C-peptide levels, were more insulin-resistant, and had higher BMI, WHR, and waist circumference values $(P<0.01)$. Simultaneously, antipsychotic-treated schizophrenia patients had far lower plasma HDL-C levels $(P<0.05)$.

Compared with antipsychotic-treated patients with schizophrenia, antipsychotic discontinuation patients with schizophrenia did not exhibit significant differences in BMI, WHR, waist circumference, and glucose and lipid metabolic parameters $(P>0.05)$.

Compared with first-episode, drug-naïve patients with schizophrenia, antipsychotic discontinuation patients had much higher plasma insulin and C-peptide levels, were much more insulin resistant, and had lower HDL-C levels $(P<0.05)$. They also had significantly larger BMI and waist circumference values $(P<0.01)$.

No significant differences in fasting plasma glucose levels among three groups were observed (see Tables 2 and 3).

\section{The relationship between $\mathrm{BMI}$, waist circumference, and plasma levels of insulin and HDL-C}

Pearson correlation analyses showed that plasma levels of insulin were positively correlated with BMI and waist circumference values in Chinese patients with schizophrenia, and plasma HDL-C levels were negatively correlated with BMI and waist circumference (see Table 4).

\section{Discussion}

This study was conducted in a natural clinical setting. The results showed that compared with drug-naïve patients with schizophrenia, antipsychotic-treated patients had significantly higher BMI, WHR, and waist circumference values and higher plasma insulin levels and insulin resistance. Additionally, their plasma HDL-C levels were lower. Our results confirm the predominant findings of previous studies, ${ }^{31-35}$ revealing a close relationship between antipsychotic drugs and glucose and lipid metabolism disorders. Indeed, reports of an association between glucose and lipid metabolism disorders and antipsychotic drugs gradually

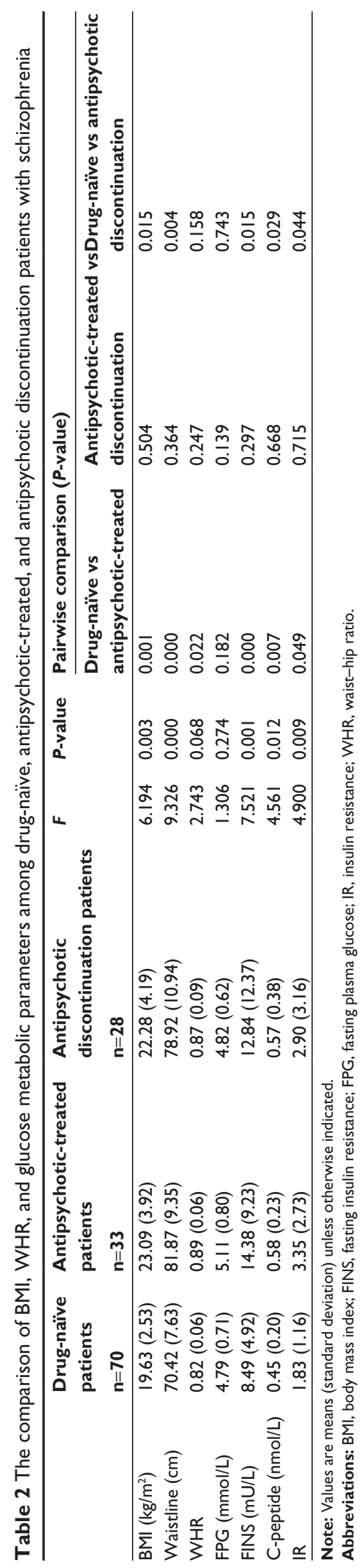




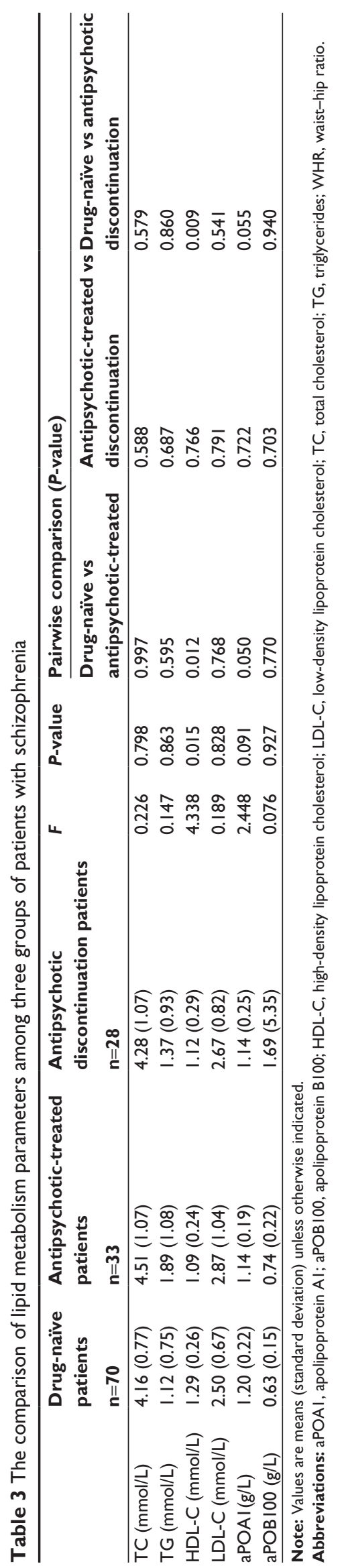

increased since chlorpromazine, the first chemically synthetic antipsychotic drug, was first used in clinical psychiatry practice ${ }^{36}$ At present, the most consistent finding across all of the relevant studies on schizophrenia is that the onset and progression of metabolism syndrome and its compound components, such as insulin resistance and lower plasma HDL-C levels, are closely correlated with the use of antipsychotic drugs. ${ }^{37}$ One related meta-analysis ${ }^{38}$ also reported that independent of whether the medication was a first- or second-generation drug, and independent of the mechanism, antipsychotic drugs had different effects on glucose and lipid metabolism disorders. The glucose and lipid metabolism disorders observed in schizophrenia patients as a result of antipsychotic drug treatment often led to significant augmentation of BMI and waist circumference values, greater insulin resistance, and much lower HDL-C plasma levels. In our previous study we also found that drug-naïve schizophrenia patients had higher fasting plasma insulin and C-peptide levels and lower fasting plasma HDL-C and aPOA1 levels and were more insulin resistant compared with healthy controls. Given the results of both the present study and our previous study, ${ }^{39}$ we can conclude that the risk of glucose and lipid metabolism disturbances among Chinese patients with schizophrenia may be associated with not only schizophrenia itself but antipsychotic drugs as well.

Furthermore, we first studied whether antipsychotic discontinuation has an evident effect on glucose and lipid metabolism in Chinese patients with schizophrenia. The results showed that compared with drug-naïve schizophrenia patients, antipsychotic discontinuation patients still had much higher BMI and waist circumference values, far higher plasma insulin levels and greater insulin resistance, and much lower plasma HDL-C levels. Additionally, compared with antipsychotic-treated schizophrenia patients, no significant improvement was found in glucose and lipid metabolic parameters among antipsychotic discontinuation patients with schizophrenia. These findings suggest that antipsychotic discontinuation appears to have no noteworthy positive effect on glucose and lipid metabolism disturbances in Chinese patients with schizophrenia. These results were assumed to be associated with three possible factors. First, the average antipsychotic discontinuation duration of 0.67 (standard deviation $=0.55$ ) years is not long enough to reverse glucose and lipid metabolism disorders resulting from antipsychotic drugs. Second, glucose and lipid metabolism disorders are, to some extent, inherent in patients with schizophrenia, ${ }^{17,18,40}$ and antipsychotic drugs may be only an 
Table 4 The relationship between BMI, waist circumference, and plasma levels of insulin and HDL-C

\begin{tabular}{lll}
\hline & Insulin $\mathbf{~ m U} / \mathbf{L}$ & HDL-C $\mathbf{~ m m o l} / \mathbf{L}$ \\
\hline BMl $\left(\mathrm{kg} / \mathrm{m}^{2}\right)$ & $r=0.256, P=0.003$ & $r=-0.303, P=0.000$ \\
Waist circumference $(\mathrm{cm})$ & $r=0.256, P=0.003$ & $r=-0.368, P=0.000$ \\
\hline
\end{tabular}

Abbreviations: BMI, body mass index; HDL-C, high-density lipoprotein cholesterol.

initiating or accelerating factor. Once initiated, glucose and lipid metabolism disturbances can develop progressively. Third, our study's sample size (28 antipsychotic discontinuation patients with schizophrenia and 33 antipsychotic-treated patients with schizophrenia) was relatively small. It may be difficult to determine differences between the treated and the discontinued group. Therefore, a similar study of larger sample size is warranted.

One previous study ${ }^{41}$ found that both BMI and waist circumference significantly predicted abnormalities in glucose homeostasis in patients with schizophrenia who took olanzapine, risperidone, ziprasidone, or first-generation antipsychotic drugs. Another study ${ }^{42}$ found that waist circumference was the best anthropometric measure for predicting obesity-related metabolic side effects such as IR that are associated with antipsychotic drugs in patients with schizophrenia. Early in 2001, the National Cholesterol Education Program Adult Treatment Panel III highlighted that waist circumference was the best surrogate for visceral adipose tissue measurements and had a strong association with cardiometabolic risk factors. ${ }^{43-46}$ Furthermore, waist circumference is a strong predictor of not only insulin resistance and type 2 diabetes ${ }^{47-49}$ but also atherogenic dyslipidemia. ${ }^{50}$ In the current study, Pearson correlation analyses of plasma levels of insulin, HDL-C, BMI, and waist circumference in all 131 Chinese patients with schizophrenia were performed, and the results showed that plasma insulin levels were positively correlated with BMI and waist circumference, whereas plasma HDL-C levels were negatively correlated with BMI and waist circumference. Our findings confirmed that insulin resistance and atherogenic dyslipidemia in patients with schizophrenia who were treated with antipsychotic drugs are closely associated with weight gain and waist circumference augmentation.

The current study has several limitations. First, its crosssectional design does not allow us to draw specific conclusions about causality. Second, we did not collect additional detailed information that could prove relevant to the results, such as smoking habits, alcohol use, diet, and exercise. A third limitation is the small sample size. Despite these limitations, our study has its merits. Firstly, to our knowledge, no previously published study has compared drug-naïve, antipsychotic-treated, and antipsychotic discontinuation patients with schizophrenia. Such a design can effectively control for the possible contribution of the illness itself to the study results. Secondly, in our analyses, we did control for primary confounding factors, including age, sex, course of illness, past medication use, and the severity of illness. Thirdly, based on retrospective interviews upon admission, we excluded treated schizophrenia patients who had possible pre-existing metabolic abnormalities. To further investigate the effects of antipsychotic drugs on glucose and lipid metabolism disorders, longitudinal studies beginning with drug-naïve schizophrenia patients should be considered and developed.

Regardless of its strengths and weaknesses, the present study carries important clinical implications. First, in the clinical practice of psychiatry, we should pay much more attention to glucose and lipid metabolism in schizophrenia patients with current or prior antipsychotic drug treatment. In particular, insulin levels, insulin resistance, and HDL-C levels should be routinely monitored. Moreover, our study results argue in favor of strengthening schizophrenia patients' compliance with antipsychotic drug treatment, because antipsychotic drug discontinuation does not significantly improve abnormalities of glucose and lipid metabolic parameters, and antipsychotic discontinuation can increase the risk of relapse or the recurrence of schizophrenia symptoms.

\section{Conclusion}

Considering all of these findings and related issues, the results of the present study suggest that antipsychotic drugs can aggravate glucose and lipid metabolism disorders; furthermore, the characteristics of glucose and lipid metabolism disorders in Chinese patients with schizophrenia who take antipsychotic drugs appear to include much higher plasma insulin levels, greater insulin resistance, and far lower plasma HDL-C levels compared with drug-naïve patients with schizophrenia. Our findings also suggest that antipsychotic discontinuation does not significantly improve parameters related to glucose and lipid metabolism disorders in Chinese patients with schizophrenia.

\section{Acknowledgments}

The authors gratefully acknowledge the contributions of all of the doctors, nurses, technicians, and subjects who participated in this study.

\section{Author contributions}

All authors contributed to the conception and design of the study, the data analysis and interpretation, drafting and revising the article for intellectual content, and final approval of the submitted version. No one who fulfilled these criteria has 
been excluded as an author. Dr Jingping Zhao accepts full responsibility for the final article.

\section{Disclosure}

This research was funded by the Science and Technology Plan Project (2009B080701080) of Guangdong Province and the National R\&D Special Fund for Health Profession (Grant No. 201002003). All of the authors met the International Committee of Medical Journal Editors (ICMJE) criteria, and all those who fulfilled those criteria are listed as authors. All authors had access to the study data, made the final decision about where to publish these data, and approved submission to this journal. The authors report no conflicts of interest in this work.

\section{References}

1. Osby U, Correia N, Brandt L, Ekbom A, et al. Mortality and causes of death in schizophrenia in Stockholm county, Sweden. Schizophr Res. 2000;45(1-2):21-28.

2. Newcomer JW. Metabolic syndrome and mental illness. Am J Manag Care. 2007;13(7 Suppl):S170-S177.

3. McEvoy JP, Meyer JM, Goff DC, et al. Prevalence of the metabolic syndrome in patients with schizophrenia: baseline results from the Clinical Antipsychotic Trials of Intervention Effectiveness (CATIE) schizophrenia trial and comparison with national estimates from NHANES III. Schizophr Res. 2005;80(1):19-32.

4. Curkendall SM, Mo J, Glasser DB, et al. Cardiovascular disease in patients with schizophrenia in Saskatchewan, Canada. J Clin Psychiatry. 2004;65(5):715-720.

5. Hennekens CH, Hennekens AR, Hollar D, et al. Schizophrenia and increased risks of cardiovascular disease. Am Heart J. 2005;150(6): $1115-1121$.

6. Wannamethee SG, Shaper AG, Lennon L, et al. Metabolic syndrome vs Framingham Risk Score for prediction of coronary heart disease, stroke, and type 2 diabetes mellitus. Arch Intern Med. 2005;165(22): 2644-2650.

7. Cohn T, Prud'homme D, Streiner D, et al. Characterizing coronary heart disease risk in chronic schizophrenia: high prevalence of the metabolic syndrome. Can J Psychiatry. 2004;49(11):753-760.

8. Ryan MC, Thakore JH. Physical consequences of schizophrenia and its treatment: the metabolic syndrome. Life Sci. 2002;71(3):239-257.

9. Bobes J, Arango C, Aranda P, et al. Cardiovascular and metabolic risk in outpatients with schizophrenia treated with antipsychotics: results of the CLAMORS study. Schizophr Res. 2007;90(1-3):162-173.

10. Melkersson K. Clozapine and olanzapine, but not conventional antipsychotics, increase insulin release in vitro. Eur Neuropsychopharmacol. 2004;14(2):115-119.

11. Smith RC, Lindenmayer JP, Bark N, et al. Clozapine, risperidone, olanzapine, and conventional antipsychotic drug effects on glucose, lipids, and leptin in schizophrenic patients. Int J Neuropsychopharmacol. 2005;8(2):183-194.

12. Koval MS, Rames LJ, Christie S. Diabetic ketoacidosis associated with clozapine treatment. Am J Psychiatry. 1994;151(10):1520-1521.

13. Mukherjee S, Schnur DB, Reddy R. Family history of type 2 diabetes in schizophrenic patients. Lancet. 1989;1(8636):495.

14. Adams PF, Marano MA. Current estimates from the National Health Interview Survey, 1994. Vital Health Stat 10. 1995;(193 Pt 1):1-260.

15. Yang J, Liu X, Liu X, et al. Abnormality of glycometabolism related factors in non-psychotic offspring of schizophrenic patients. Psychiatry Res. 2012;198(2):183-186.
16. Spelman LM, Walsh PI, Sharifi N, et al. Impaired glucose tolerance in first-episode drug-naive patients with schizophrenia. Diabet Med. 2007;24(5):481-485.

17. Guest PC, Wang L, Harris LW, et al. Increased levels of circulating insulin-related peptides in first-onset, antipsychotic naive schizophrenia patients. Mol Psychiatry. 2010;15(2):118-119.

18. Dasgupta A, Singh OP, Rout JK, et al. Insulin resistance and metabolic profile in antipsychotic naive schizophrenia patients. Prog Neuropsychopharmacol Biol Psychiatry. 2010;34(7):1202-1207.

19. Freeman H. Resistance to insulin in mentally disturbed soldiers. Arch Neurol Psychiatry. 1946;56(1):74-78.

20. Liebzeit KA, Markowitz JS, Caley CF. New onset diabetes and atypical antipsychotics. Eur Neuropsychopharmacol. 2001;11(1):25-32.

21. Koller E, Schneider B, Bennett K, et al. Clozapine-associated diabetes. Am J Med. 2001;111(9):716-723.

22. Melkersson KI, Dahl ML, Hulting AL. Guidelines for prevention and treatment of adverse effects of antipsychotic drugs on glucoseinsulin homeostasis and lipid metabolism. Psychopharmacology (Berl). 2004;175(1):1-6.

23. Leslie DL, Rosenheck RA. Incidence of newly diagnosed diabetes attributable to atypical antipsychotic medications. Am J Psychiatry. 2004;161(9):1709-1711.

24. Mackin P, Bishop D, Watkinson H, et al. Metabolic disease and cardiovascular risk in people treated with antipsychotics in the community. Br J Psychiatry. 2007;191:23-29.

25. Birkenaes AB, Birkeland KI, Engh JA, et al. Dyslipidemia independent of body mass in antipsychotic-treated patients under real-life conditions. J Clin Psychopharmacol. 2008;28(2):132-137.

26. Patel NC, Hariparsad M, Matias-Akthar M, et al. Body mass indexes and lipid profiles in hospitalized children and adolescents exposed to atypical antipsychotics. J Child Adolesc Psychopharmacol. 2007;17(3):303-311.

27. Gaulin BD, Markowitz JS, Caley CF, et al. Clozapine-associated elevation in serum triglycerides. Am J Psychiatry. 1999;156(8): 1270-1272.

28. Leitao-Azevedo CL, Guimaraes LR, de Abreu MG, et al. Increased dyslipidemia in schizophrenic outpatients using new generation antipsychotics. Rev Bras Psiquiatr. 2006;28(4):301-304.

29. Haffner SM, Miettinen H, Stern MP. The homeostasis model in the San Antonio Heart Study. Diabetes Care. 1997;20(7):1087-1092.

30. Friedewald WT, Levy RI, Fredrickson DS. Estimation of the concentration of low-density lipoprotein cholesterol in plasma, without use of the preparative ultracentrifuge. Clin Chem. 1972;18(6):499-502.

31. Tirupati S, Chua LE. Obesity and metabolic syndrome in a psychiatric rehabilitation service. Aust N Z J Psychiatry. 2007;41(7):606-610.

32. Wang G, Li YF. [Association between lipoprotein(a) and coronary artery disease]. Zhonghua Xin Xue Guan Bing Za Zhi. 2010;38(7):618-620.

33. Patel JK, Buckley PF, Woolson S, et al. Metabolic profiles of secondgeneration antipsychotics in early psychosis: findings from the CAFE study. Schizophr Res. 2009;111(1-3):9-16.

34. Perez-Iglesias R, Crespo-Facorro B, Amado JA, et al. A 12-week randomized clinical trial to evaluate metabolic changes in drug-naive, first-episode psychosis patients treated with haloperidol, olanzapine, or risperidone. J Clin Psychiatry. 2007;68(11):1733-1740.

35. Zipursky RB, Gu H, Green AI, et al. Course and predictors of weight gain in people with first-episode psychosis treated with olanzapine or haloperidol. Br J Psychiatry. 2005;187:537-543.

36. Ryan MM, Huffaker SJ, Webster MJ, et al. Application and optimization of microarray technologies for human postmortem brain studies. Biol Psychiatry. 2004;55(4):329-336.

37. Yogaratnam J, Biswas N, Vadivel R, et al. Metabolic complications of schizophrenia and antipsychotic medications-an updated review. East Asian Arch Psychiatry. 2013;23(1):21-28.

38. Chadda RK, Ramshankar P, Deb KS, et al. Metabolic syndrome in schizophrenia: differences between antipsychotic-naive and treated patients. J Pharmacol Pharmacother. 2013;4(3):176-186. 
39. Wu X, Huang $\mathrm{Z}$, Wu R, et al. The comparison of glycometabolism parameters and lipid profiles between drug-naive, first-episode schizophrenia patients and healthy controls. Schizophr Res. 2013;150(1): $157-162$.

40. Reaven GM. Banting lecture 1988. Role of insulin resistance in human disease. Diabetes. 1988;37(12):1595-1607.

41. Haupt DW, Fahnestock PA, Flavin KA, et al. Adiposity and insulin sensitivity derived from intravenous glucose tolerance tests in antipsychotic-treated patients. Neuropsychopharmacology. 2007;32(12): 2561-2569.

42. Henderson DC, Fan X, Sharma B, et al. Waist circumference is the best anthropometric predictor for insulin resistance in nondiabetic patients with schizophrenia treated with clozapine but not olanzapine. J Psychiatr Pract. 2009;15(4):251-261.

43. Onat A, Avci GS, Barlan MM, et al. Measures of abdominal obesity assessed for visceral adiposity and relation to coronary risk. Int J Obes Relat Metab Disord. 2004;28(8):1018-1025.

44. Jia WP, Lu JX, Xiang KS, et al. Prediction of abdominal visceral obesity from body mass index, waist circumference and waist-hip ratio in Chinese adults: receiver operating characteristic curves analysis. Biomed Environ Sci. 2003;16(3):206-211.
45. Shen W, Punyanitya M, Chen J, et al. Waist circumference correlates with metabolic syndrome indicators better than percentage fat. Obesity (Silver Spring). 2006;14(4):727-736.

46. Ho SC, Chen YM, Woo JL, et al. Association between simple anthropometric indices and cardiovascular risk factors. Int JObes Relat Metab Disord. 2001;25(11):1689-1697.

47. Farin HM, Abbasi F, Reaven GM. Body mass index and waist circumference correlate to the same degree with insulin-mediated glucose uptake. Metabolism. 2005;54(10):1323-1328.

48. Farin HM, Abbasi F, Reaven GM. Body mass index and waist circumference both contribute to differences in insulin-mediated glucose disposal in nondiabetic adults. Am J Clin Nutr. 2006;83(1):47-51.

49. Farin HM, Abbasi F, Reaven GM. Comparison of body mass index versus waist circumference with the metabolic changes that increase the risk of cardiovascular disease in insulin-resistant individuals. $\mathrm{Am}$ $J$ Cardiol. 2006;98(8):1053-1056.

50. Dalton M, Cameron AJ, Zimmet PZ, et al. Waist circumference, waisthip ratio and body mass index and their correlation with cardiovascular disease risk factors in Australian adults. J Intern Med. 2003;254(6): $555-563$.
Neuropsychiatric Disease and Treatment

\section{Publish your work in this journal}

Neuropsychiatric Disease and Treatment is an international, peerreviewed journal of clinical therapeutics and pharmacology focusing on concise rapid reporting of clinical or pre-clinical studies on a range of neuropsychiatric and neurological disorders. This journal is indexed on PubMed Central, the 'PsycINFO' database and CAS,

\section{Dovepress}

and is the official journal of The International Neuropsychiatric Association (INA). The manuscript management system is completely online and includes a very quick and fair peer-review system, which is all easy to use. Visit http://www.dovepress.com/testimonials.php to read real quotes from published authors. 